\title{
(GIGA)bYte
}

\section{Annotation of chitin biosynthesis genes in Diaphorina citri, the Asian citrus psyllid}

\author{
Sherry Miller ${ }^{1,2}$, Teresa D. Shippy ${ }^{1}$, Blessy Tamayo ${ }^{3}$, Prashant S. Hosmani ${ }^{4}$, \\ Mirella Flores-Gonzalez ${ }^{4}$, Lukas A. Mueller ${ }^{4}$, Wayne B. Hunter ${ }^{5}$, \\ Susan J. Brown ${ }^{1}$, Tom D’Elia ${ }^{3}$ and Surya Saha ${ }^{4,6, *}$ \\ 1 Division of Biology, Kansas State University, Manhattan, KS 66506, USA \\ 2 Allen County Community College, Burlingame, KS 66413, USA \\ 3 Indian River State College, Fort Pierce, FL 34981, USA \\ 4 Boyce Thompson Institute, Ithaca, NY 14853, USA \\ 5 USDA-ARS, U.S. Horticultural Research Laboratory, Fort Pierce, FL 34945, USA \\ 6 Animal and Comparative Biomedical Sciences, University of Arizona, Tucson, AZ 85721, USA
}

\section{ABSTRACT}

The polysaccharide chitin is critical for the formation of many insect structures, including the exoskeleton, and is required for normal development. Here we report the annotation of three genes from the chitin synthesis pathway in the Asian citrus psyllid, Diaphorina citri (Hemiptera: Liviidae), the vector of Huanglongbing (citrus greening disease). Most insects have two chitin synthase (CHS) genes but, like other hemipterans, D. citri has only one. In contrast, D. citri is unusual among insects in having two UDP-N-acetylglucosamine pyrophosphorylase (UAP) genes. One of the $D$. citri UAP genes is broadly expressed, while the other is expressed predominantly in males. Our work helps pave the way for potential utilization of these genes as pest control targets to reduce the spread of Huanglongbing.

Subjects Genetics and Genomics, Animal Genetics, Bioinformatics

Submitted: Accepted:

19 December 2020

Published:

03 June 2021

10 June 2021

* Corresponding author. E-mail: suryasaha@cornell.edu

Published by GigaScience Press.

Preprint submitted at https:

//doi.org/10.1101/2020.09.22.309211

This is an Open Access article distributed under the terms of the Creative Commons Attribution License (http://creativecommons.org/ licenses/by/4.0/), which permits unrestricted reuse, distribution, and reproduction in any medium, provided the original work is properly cited.

Gigabyte, 2021, 1-12

\section{DATA DESCRIPTION}

\section{Introduction}

Chitin is a polysaccharide that is essential for insect development. It is crucial in the development of the insect cuticle and exoskeleton, the peritrophic membrane of the midgut of some insects, and other structures such as the trachea, wing hinges and eggshell [1]. Because chitin is essential for insect development but is not found in mammals, the enzymes involved in its synthesis are considered attractive targets for pest control. The biosynthetic pathway for chitin begins with the hexosamine pathway, in which simple sugars, such as glucose, trehalose and glycogen, are converted into UDP-N-acetylglucosamine (UDP-GlcNAc). The final step in the hexosamine pathway is catalyzed by the enzyme UDP-N-acetylglucosamine pyrophosphorylase (UAP) [1]. UDP-GlcNAc is then converted to chitin by enzymes known as chitin synthases (CHS) [1].

\section{Context}

Here we report the annotation of the CHS and UAP genes in genome version 3 (v3) of the Asian citrus psyllid, Diaphorina citri (Hemiptera: Liviidae; NCBI:txid121845), the vector for 


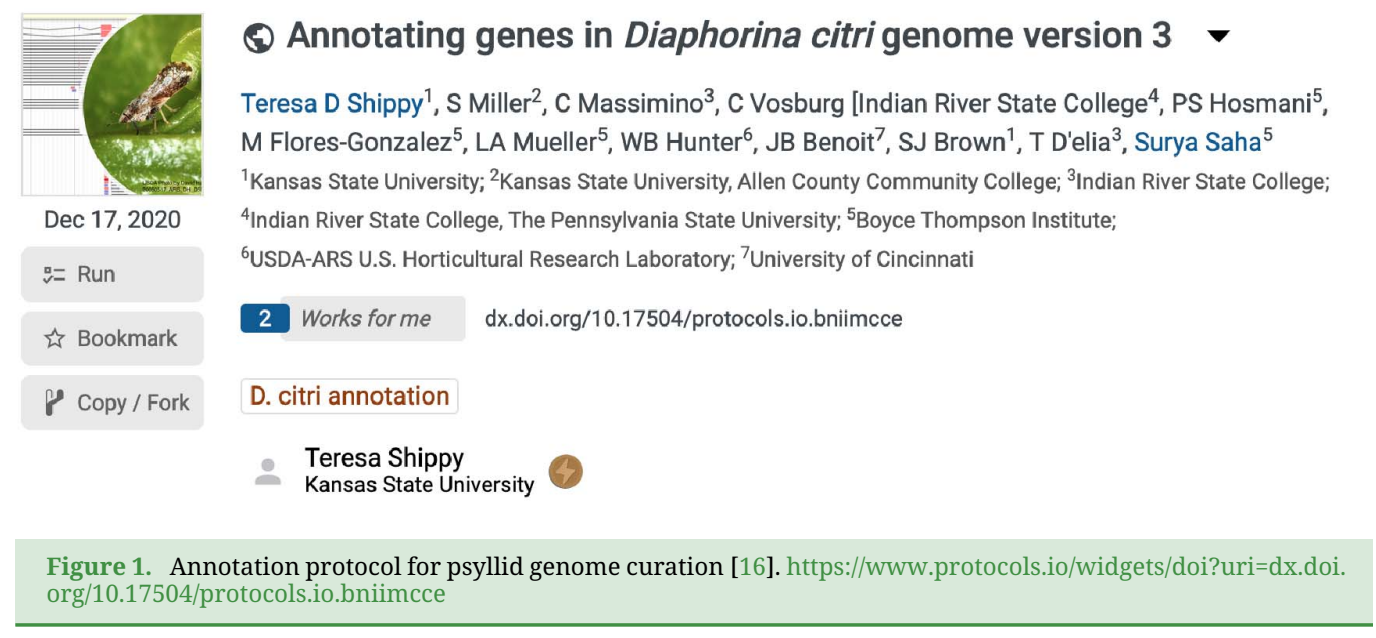

\begin{tabular}{|c|c|c|c|c|c|c|c|c|c|}
\hline & $\begin{array}{c}\text { Drosophila } \\
\text { melanogaster }\end{array}$ & $\begin{array}{c}\text { Anopheles } \\
\text { gambiae }\end{array}$ & $\begin{array}{c}\text { Aedes } \\
\text { aegypti }\end{array}$ & $\begin{array}{c}\text { Tribolium } \\
\text { castaneum }\end{array}$ & $\begin{array}{c}\text { Apis } \\
\text { mellifera }\end{array}$ & $\begin{array}{c}\text { Nasonia } \\
\text { vitripennis }\end{array}$ & $\begin{array}{c}\text { Acyrthosiphon } \\
\text { pisum }\end{array}$ & $\begin{array}{c}\text { Bemisia } \\
\text { tabaci }\end{array}$ & $\begin{array}{l}\text { Diaphorina } \\
\text { citri }\end{array}$ \\
\hline CHS1/A & 1 & 1 & 1 & 1 & 1 & 1 & 1 & 1 & 1 \\
\hline CHS2/B & 1 & 1 & 1 & 1 & 1 & 1 & 0 & 0 & 0 \\
\hline UAP & 1 & 1 & 1 & 2 & 1 & 1 & 1 & 1 & 2 \\
\hline
\end{tabular}

the bacterium that causes Huanglongbing (citrus greening disease). The $D$. citri v3 genome is a chromosome-level assembly with a 40.5-megabase pair (Mb) scaffold N50 value, and 88.3\% complete Benchmarking Universal Single-Copy Orthologs (BUSCO) [2]. However, due to heterogeneity of the sequenced psyllids, the genome has numerous false duplications of varying sizes, ranging from multiple adjacent genes to partial exons. As with all genomes, computationally annotated models provide a starting point, but often require manual correction.

We identified and manually annotated one CHS gene and two UAP genes in the $D$. citri genome v3. Although most insects have two CHS genes [3, 4] (Table 1), the presence of a single CHS gene is consistent with reports from other hemipteran genomes [5]. In contrast, $D$. citri seems to be unusual in that it has two UAP genes. Available RNA-seq data indicate that one of the $D$. citri UAP genes is broadly expressed, while the other is expressed predominantly in males. Our manual annotation of these chitin biosynthesis genes provides more accurate information for the design of future experiments involving these genes.

\section{METHODS}

D. citri genes in genome v3 [2] were identified by BLAST (NCBI BLAST, RRID:SCR_004870) analysis of $D$. citri sequences with insect CHS and UAP orthologs. Reciprocal BLAST of the National Center for Biotechnology Information (NCBI) non-redundant protein database [15] was used to confirm orthology. Manual annotation of genes was performed in Apollo (Apollo, RRID:SCR_001936; v2.1.0) using RNA-seq reads, Iso-seq transcripts and de novo-assembled transcripts as evidence. A more detailed description of the annotation workflow is available via protocols.io (Figure 1) [16]. 


\begin{tabular}{|c|c|c|c|}
\hline Species & Accession & Name in NCBI & Name in Tree \\
\hline Tribolium castaneum & NP_001034491.1 & chitin synthase 1 & Tc CHS1 \\
\hline Anopheles gambiae & XP_321336.5 & AGAP001748-PA & Ag CHS1 \\
\hline Apis mellifera & XP_016770736.1 & $\begin{array}{l}\text { PREDICTED: uncharacterized protein LOC412215 } \\
\text { isoform X1 }\end{array}$ & Am LOC412215 \\
\hline Nasonia vitripennis & XP_008215129.1 & $\begin{array}{l}\text { PREDICTED: uncharacterized protein LOC100118280 } \\
\text { isoform X1 }\end{array}$ & Nv LOC100118280 \\
\hline Acyrthosiphon pisum & XP_003247517.1 & PREDICTED: uncharacterized protein LOC100162079 & Ap LOC100162079 \\
\hline Bemisia tabaci & XP_018916997.1 & $\begin{array}{l}\text { PREDICTED: uncharacterized protein LOC109044007 } \\
\text { isoform X1 }\end{array}$ & Bt LOC109044007 \\
\hline Drosophila melanogaster & NP_524233.1 & krotzkopf verkehrt, isoform A & Dm krotzkopf verkehrt \\
\hline Manduca sexta & AAL38051.2 & chitin synthase & Ms CHS1 \\
\hline Spodoptera exigua & AAZ03545.1 & chitin synthase A & Se CHSA \\
\hline Tribolium castaneum & NP_001034492.1 & chitin synthase 2 & Tc CHS2 \\
\hline Manduca sexta & AAX20091.1 & chitin synthase 2 & Ms CHS2 \\
\hline Spodoptera exigua & ABI96087.1 & chitin synthase B & Se CHSB \\
\hline Drosophila melanogaster & NP_524209.3 & chitin synthase 2 & Dm CHS2 \\
\hline Anopheles gambiae & XP_321951.2 & AGAP001205-PA & Ag CHS2 \\
\hline Apis mellifera & XP_016767448.1 & chitin synthase chs-2 & Am CHS-2 \\
\hline Nasonia vitripennis & XP_008215122.2 & chitin synthase chs-2 & Nv CHS-2 \\
\hline Drosophila melanogaster & NP_001285673.1 & mummy, isoform D & Dm Mummy \\
\hline Anopheles gambiae & XP_317600.4 & AGAP007889-PA & Ag UAP \\
\hline Aedes aegypti & EAT47260.1 & AAEL001627-PA & Aa UAP \\
\hline Bombyx mori & NP_001296486.1 & $\begin{array}{l}\text { UDP-N-acetylhexosamine pyrophosphorylase-like } \\
\text { protein } 1\end{array}$ & Bm UAP \\
\hline Tribolium castaneum & NP_001164533.1 & UDP-N-acetylglucosamine pyrophosphorylase 1 & Tc UAP1 \\
\hline Tribolium castaneum & NP_001164534.1 & UDP-N-acetylglucosamine pyrophosphorylase 2 & Tc UAP2 \\
\hline Apis mellifera & XP_624349.1 & UDP-N-acetylhexosamine pyrophosphorylase & Am UAP \\
\hline Nasonia vitripennis & XP_001602623.1 & UDP-N-acetylhexosamine pyrophosphorylase & Nv UAP \\
\hline Acyrthosiphon pisum & XP_001944680.1 & UDP-N-acetylhexosamine pyrophosphorylase & Ap UAP \\
\hline Bemisia tabaci & XP_018902053.1 & $\begin{array}{l}\text { PREDICTED: UDP-N-acetylhexosamine } \\
\text { pyrophosphorylase }\end{array}$ & Bt UAP \\
\hline Locusta migratoria & AGN56418.1 & UDP N-acetylglucosamine pyrophosphorylases 1 & Lm UAP1 \\
\hline Locusta migratoria & AGN56419.1 & UDP N-acetylglucosamine pyrophosphorylases 2 & Lm UAP2 \\
\hline Leptinotarsa decemlineata & XP_023024177.1 & UDP-N-acetylhexosamine pyrophosphorylase-like & Ld UAP1 \\
\hline Leptinotarsa decemlineata & XP_023022882.1 & $\begin{array}{l}\text { UDP-N-acetylhexosamine pyrophosphorylase-like } \\
\text { protein } 1\end{array}$ & Ld UAP2 \\
\hline
\end{tabular}

Multiple alignments of the predicted $D$. citri proteins and their insect homologs were performed using MUSCLE (RRID:SCR_011812) [17] or CLUSTALW (RRID:SCR_002909) [18] within MEGAX (MEGA software, RRID:SCR_000667), as specified in each figure legend. Phylogenetic trees were constructed using full-length protein sequences in MEGAX. Orthologs used in tree construction are listed in Table 2. Gene expression levels (Table 3) were obtained from the Citrus Greening Expression Network [19] and visualized using Excel (Microsoft Excel, RRID:SCR_016137) and the pheatmap package (pheatmap, RRID:SCR_016418) in R (R Project for Statistical Computing, RRID:SCR_001905) [20, 21].

\section{DATA VALIDATION AND QUALITY CONTROL}

\section{Chitin synthases}

Chitin synthases are the only enzymes in the chitin biosynthetic pathway that act specifically in the synthesis of chitin. This makes them an attractive, insect-specific target for RNA interference (RNAi)-based insecticides. The two CHS genes found in most 


\begin{tabular}{|c|c|c|c|c|}
\hline Gene/Transcript name & CHS-RA & CHS-RB & UAP1 & UAP2 \\
\hline Gene ID & Dcitr04g09970.1.1 & Dcitr04g09970.1.2 & Dcitr08g04630.1.1 & Dcitr05g05060.1.1 \\
\hline Egg Citrus macrophylla CLas- Whole body & 29.67 & 5.79 & 76.03 & 0.28 \\
\hline Nymph Citrus medica CLas+ Low infection Whole body & 28.07 & 50.83 & 53.3 & 3.04 \\
\hline Nymph Citrus sinensis CLas+ High infection Whole body & 18.9 & 57.96 & 48.58 & 2.89 \\
\hline Nymph $C$. sinensis CLas- Whole body & 10.8 & 57.65 & 43.84 & 2.25 \\
\hline Nymph C. macrophylla CLas- Whole body & 51.71 & 20.61 & 22.3 & 2.3 \\
\hline Nymph Citrus spp. CLas- Whole body & 21.04 & 0 & 24.12 & 0.17 \\
\hline Nymph Citrus spp. CLas+ Whole body & 16.14 & 0 & 112.11 & 3.96 \\
\hline Adult $C$. medica $C$ Las- Gut & 0.21 & 0 & 16.28 & 1.41 \\
\hline Adult $C$. medica $C$ Las+ Gut & 0.04 & 0.01 & 15.36 & 0.53 \\
\hline Adult $C$. medica $C$ Las+ High infection Whole body & 8.52 & 2 & 18.82 & 24.16 \\
\hline Adult $C$. medica CLas+ Low infection Whole body & 6.67 & 7.11 & 22.09 & 26.83 \\
\hline Adult $C$. medica $C$ Las- Whole body & 14.39 & 22.71 & 25.51 & 17.25 \\
\hline Adult C. macrophylla CLas-Whole body & 0.51 & 0 & 26.1 & 48.95 \\
\hline Adult Citrus spp. CLas- Whole body & 0.19 & 0 & 12.56 & 40.68 \\
\hline Adult Citrus spp. CLas+ Whole body & 0.41 & 0 & 29.15 & 18.13 \\
\hline Adult Citrus spp. CLas- midgut & 0.15 & 0 & 28.82 & 1.12 \\
\hline Adult Citrus spp. CLas + midgut & 0.69 & 0 & 20.8 & 5.57 \\
\hline Adult Citrus reticulata CLas- Female abdomen & 0.44 & 0 & 72.64 & 0.5 \\
\hline Adult $C$. reticulata CLas- Female antennae & 0.65 & 0.09 & 70.19 & 1.59 \\
\hline Adult $C$. reticulata $C$ Las- Female head & 0.73 & 0 & 73.58 & 0.09 \\
\hline Adult $C$. reticulata CLas- Female leg & 0.41 & 0 & 109.73 & 0 \\
\hline Adult $C$. reticulata $C$ Las- Female terminal abdomen & 1.01 & 0 & 149.58 & 1.03 \\
\hline Adult C. reticulata CLas- Female thorax & 0.49 & 0 & 40.29 & 0.28 \\
\hline Adult $C$. reticulata $C$ Las- Male abdomen & 0.35 & 0 & 50.21 & 34.24 \\
\hline Adult $C$. reticulata $C$ Las- Male antennae & 1.17 & 0.13 & 56.8 & 10.87 \\
\hline Adult $C$. reticulata $C$ Las- Male head & 0.77 & 0 & 59.63 & 0.29 \\
\hline Adult C. reticulata CLas- Male leg & 0.12 & 0 & 55.29 & 12.29 \\
\hline Adult $C$. reticulata $C$ Las- Male terminal abdomen & 0.96 & 0 & 92.77 & 19.86 \\
\hline Adult $C$. reticulata CLas-Male thorax & 0.25 & 0 & 31.74 & 2.03 \\
\hline Adult $C$. reticulata CLas- Female antennae [22] & 1.41 & 0.44 & 27.94 & 0.03 \\
\hline Adult $C$. reticulata CLas- Female terminal abdomen [22] & 0.32 & 0 & 44.29 & 0.99 \\
\hline Adult $C$. reticulata CLas- Male antennae [22] & 3.68 & 0.44 & 27.89 & 5.05 \\
\hline Adult $C$. reticulata CLas- Male terminal abdomen [22] & 0.59 & 0 & 38.01 & 39.26 \\
\hline \multicolumn{5}{|c|}{$\begin{array}{l}\text { CHS-RA: Chitin synthase-RA; CHS-RB: Chitin synthase-RB; CLas: Candidatus Liberibacter asiaticus; UAP1: UDP-N-acetylglucosamine pyrophosphorylase 1; UAP2: } \\
\text { UDP-N-acetylglucosamine pyrophosphorylase } 2 \text {. TPM values for annotated chitin biosynthesis genes from available RNA-seq experiments. All data is publicly } \\
\text { available and was obtained from the Citrus Greening Expression Network (CGEN) [19]. For each sample, information on developmental stage, food source, CLas } \\
\text { infection status and tissue are provided in the first column. }\end{array}$} \\
\hline
\end{tabular}

holometabolous insects have distinct functions. $C H S 1$, also referred to as $C H S A$, produces the chitin essential for proper cuticle development [4, 23, 24]. CHS2, also referred to as CHSB, is not required for cuticle development, but is instead essential for proper development of the gut peritrophic membrane [4, 23, 24]. RNAi knockdown of either $C H S$ gene is lethal in holometabolous insects [25-28].

Previous searches of the Acyrthosiphon pisum, Nilaparvata lugens and Rhodnius prolixus genomes identified $C H S 1$ but not $C H S 2$, suggesting that $C H S 2$ has probably been lost in the hemipteran lineage [5]. Loss of the chitin synthase gene required for peritrophic membrane development is not particularly surprising, since hemipterans do not have peritrophic membranes [5, 29]. Lu et al. [30] identified a D. citri CHS gene that clustered with other hemipteran $\mathrm{CHS}$ genes and was expressed at high levels in most adult body tissues, but at low levels in midgut, as would be expected for a CHS1 gene. Two groups have shown that 


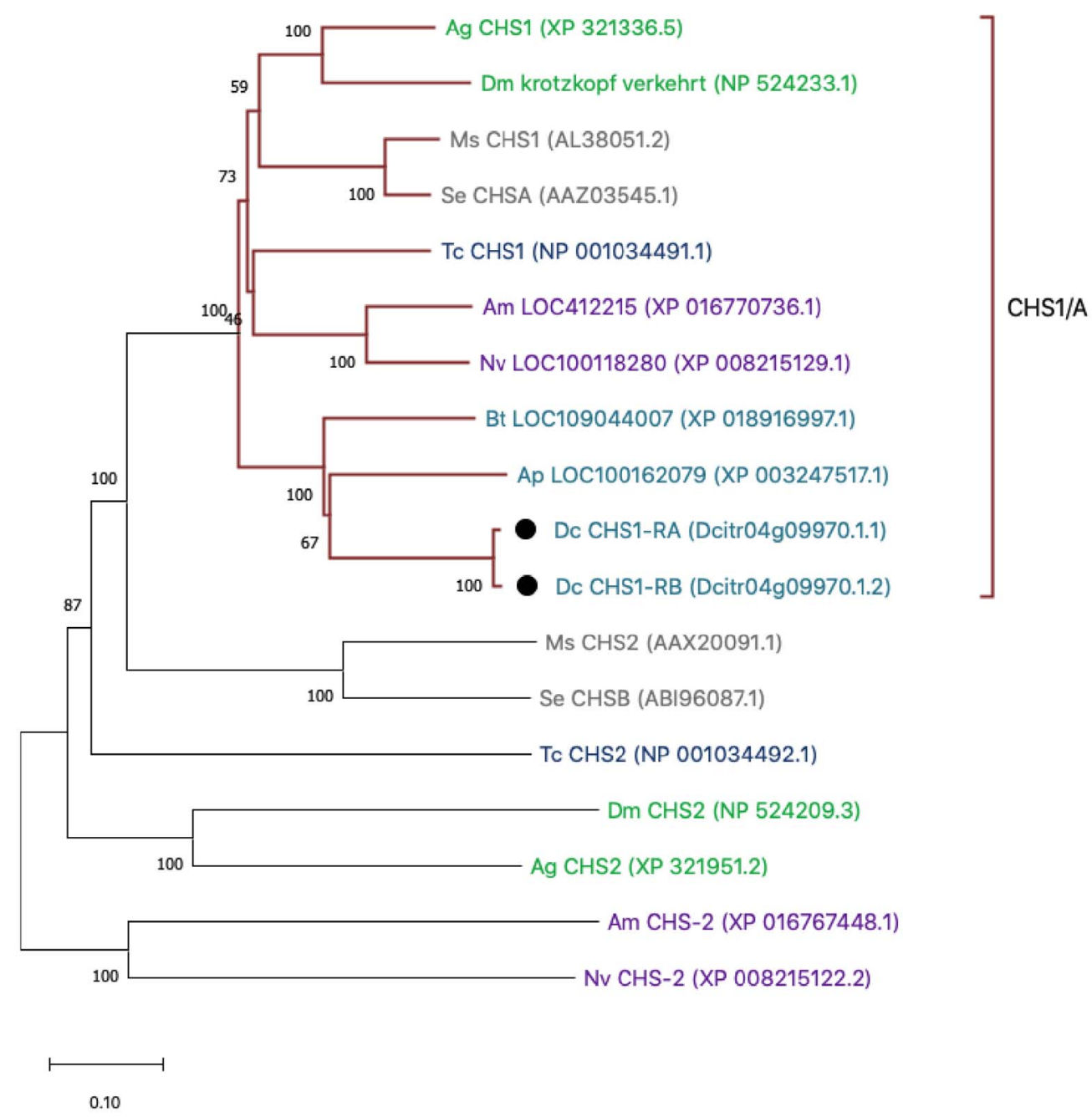

Figure 2. Phylogenetic analysis of insect CHS proteins. Species represented are Drosophila melanogaster (Dm), Anopheles gambiae (Ag), Tribolium castnaeum (Tc), Manduca sexta (Ms), Spodoptera exigua (Se), Apis mellifera (Am), Nasonia vitripennis (Nv), Acyrthosiphon pisum (Ap), Bemisia tabaci (Bt) and Diaphorina citri (Dc). MUSCLE (RRID:SCR_011812) [31] software was used to perform multiple sequence alignments of full-length protein sequences and the tree was constructed with MEGAX (RRID:SCR_000667) [32] software using the neighbor-joining method with 100 bootstrap replications. The maroon clade shows monophyletic clustering of CHS1/A genes. With the exception of $D$. citri (denoted by black circles), only one isoform per species is depicted. Taxon name color represents insect order: Diptera (green), Coleoptera (navy), Hymenoptera (purple), Lepidoptera (gray), and Hemiptera (teal).

RNAi knockdown of CHS in D. citri causes increased lethality [30, 33], supporting the idea that this gene is a good target for pest control.

Our searches of the $D$. citri v3 genome revealed the previously described $C H S$ gene, but no additional chitin synthase orthologs (Table 1). Transcriptomic evidence supports the existence of two CHS isoforms (Table 4) that differ only in the use of one alternative exon and produce proteins with slightly different C-termini. Similar isoforms of CHS1/A have been described in other insects [3,34,35]. Both isoforms of $D$. citri CHS cluster in a monophyletic clade with CHS1 proteins from other insects (Figure 2), so we have named this gene CHS1. We retrieved expression data for both isoforms of CHS1 from the Citrus 


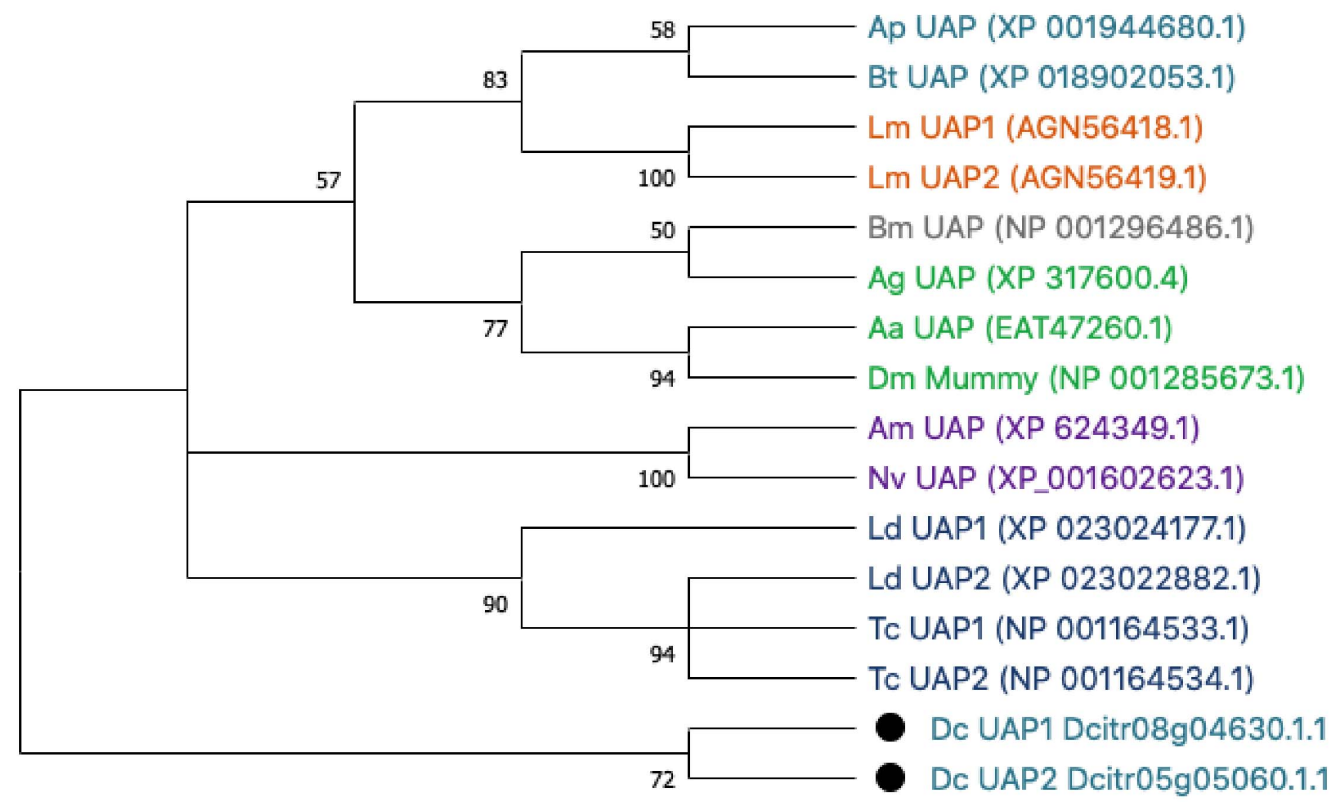

Figure 3. Phylogenetic analysis of representative insect UAP orthologs. Species shown are Drosophila melanogaster (Dm), Anopheles gambiae (Ag), Aedes aegypti (Aa), Bombyx mori (Bm), Tribolium castaneum (Tc), Leptinotarsa decemlineata (Ld), Apis mellifera (Am), Nasonia vitripennis (Nv), Locusta migratoria (Lm), Acyrthosiphon pisum (Ap), Bemisia tabaci (Bt) and Diaphorina citri (Dc and black circles). ClustalW software was used to perform the multiple sequence alignment of full-length protein sequences and a bootstrap consensus tree was constructed with MEGA X software using the neighbor-joining method with 100 bootstrap replications. Colors denote insect orders: Hemiptera (teal), Orthoptera (orange), Lepidoptera (gray), Diptera (green), Hymenoptera (purple) and Coleoptera (navy).

\begin{tabular}{|c|c|c|c|c|c|c|}
\hline \multirow[t]{2}{*}{ Gene/Isoform } & \multirow[t]{2}{*}{ OGSv3 ID } & \multirow{2}{*}{$\begin{array}{l}\text { Gene model } \\
\text { Complete }\end{array}$} & \multicolumn{4}{|c|}{ Evidence supporting annotation } \\
\hline & & & Mсот & Iso-seq & RNA-seq & Ortholog \\
\hline \multirow[t]{2}{*}{ CHS1 } & Dcitr04g09970.1.1 & $\mathrm{X}$ & MCOT15276.0.CT & $\mathrm{X}$ & $\mathrm{X}$ & $\mathrm{X}$ \\
\hline & Dcitr04g09970.1.2 & & MCOT13830.0.CO & & & \\
\hline UAP1 & Dcitr08g04630.1.1 & $\mathrm{X}$ & & $\mathrm{X}$ & & $\mathrm{X}$ \\
\hline UAP2 & Dcitr05g05060.1.1 & $\mathrm{X}$ & & $\mathrm{X}$ & $\mathrm{X}$ & $\mathrm{x}$ \\
\hline $\begin{array}{l}\text { MCOT: MAKER } \\
\text { RRID:SCR_0118 } \\
\text { assigned an OG } \\
\text { Evidence types } \\
\text { and their stren }\end{array}$ & $\begin{array}{l}\text { MAKER, RRID:SCR } \\
\text { 6), Trinity (Trinity, } \\
\text { v3 gene identifier a } \\
\text { lsed for manual ann } \\
\text { ths and weaknesses }\end{array}$ & $\begin{array}{l}\text { 5309), Cufflin } \\
\text { RID:SCR_0130 } \\
\text { denoted as a } 1 \\
\text { ation are shou } \\
\text { included in ou }\end{array}$ & $\begin{array}{l}\text { (Cufflinks, RRID: } \\
\text { ) pipeline. Each m } \\
\text { rtial or complete m } \\
\text { for each gene. A d } \\
\text { online protocol [16 }\end{array}$ & $\begin{array}{l}\text { ally ann } \\
\text { el based c } \\
\text { ription of }\end{array}$ & $\begin{array}{l}\text { ses (Oases, } \\
\text { ed gene has } \\
\text { vailable ev } \\
\text { various ev }\end{array}$ & $\begin{array}{l}\text { nce. } \\
\text { nce sources }\end{array}$ \\
\hline
\end{tabular}

Greening Expression Network (CGEN), which contains RNA-seq data sets for various life stages and tissues [19]. Data from whole body samples indicate that CHS1 is expressed at all life stages, but is most highly expressed in juvenile stages (Figure 4).

Our manual annotation of CHS1 corrects several errors that were present in the previous computationally predicted annotation for D. citri CHS (XP_017303059). Changes to the model include the addition of formerly missing sequence and the removal of artifactually duplicated regions. Domain analysis with TMHMM Server (TMHMM Server, RRID:SCR_014935, v2.0) indicates that the corrected CHS1-RA and CHS1-RB proteins have 15 transmembrane helices, as is typical for insect CHS proteins, rather than the 14 that were reported for the earlier version of the protein [30]. 


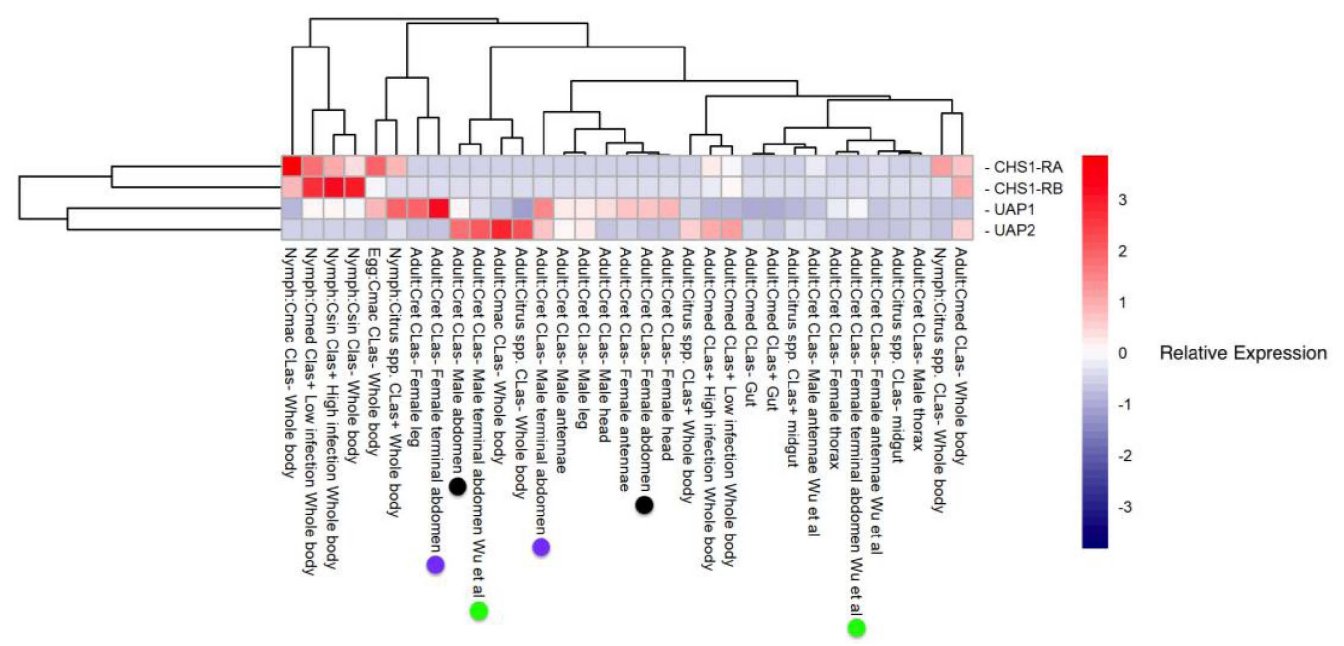

Figure 4. Heatmap representation of chitin biosynthesis gene expression levels in various RNA-seq datasets. Expression levels were obtained as transcripts per million (TPM) from the Citrus Greening Expression Network [19] and the heatmap was scaled by row. For ease of comparison, colored circles denote pairs of male and female abdominal tissue samples from the same experiments.

\section{UDP-N-acetylglucosamine pyrophosphorylase (UAP)}

In addition to its role in chitin synthesis, UAP is involved in the modification of other carbohydrates, sphingolipids and proteins. In Drosophila, mutants of UAP (also called mummy, cabrio and cystic) have defects in tracheal development, dorsal closure, eye development and nervous system function [36-38]. Some of these developmental defects are caused by disruption of the chitin synthesis pathway, while others appear to be caused by effects on other glycoproteins. For example, defects in embryonic dorsal closure have been linked to a role for UAP in regulation of Decapentaplegic signaling [6].

Most insects appear to have a single UAP gene (Table 1) [39]. However, a few insects, including T. castaneum, Locusta migratoria and Leptinotarsa decemlineata have two UAP genes [39-41]. Comparison of the T. castaneum and L. migratoria gene pairs indicates that they arose through separate, relatively recent lineage-specific gene duplications [40]. RNAi experiments in T. castaneum showed that UAP1 is involved in the biosynthesis of chitin both in the cuticle and the peritrophic membrane, while UAP2 is important for the modification of other macromolecules [39]. In L. migratoria, LmUAP1 knockdown caused lethality and defects consistent with disruption of chitin biosynthesis, while LmUAP2 knockdown did not increase lethality and produced no visible effects [40].

In the $D$. citri v3 genome, we identified two UAP genes located on different chromosome-length scaffolds. The proteins encoded by these apparent paralogs share $50 \%$ identity, distributed throughout the length of the proteins (Figure 5), which is similar to the level of identity shared with UAP orthologs from closely related insect species. Amino acid residues known to be important for substrate binding in the human UAP ortholog and conserved in the T. castaneum UAP proteins [39] are also well conserved in the D. citri UAP proteins (Figure 5). Phylogenetic analysis (Figure 3) suggests that the two genes represent a lineage-specific duplication. Surprisingly, the $D$. citri UAP proteins do not cluster with the other hemipteran UAP proteins; instead, they appear as an outgroup to all the other insect UAP proteins. This suggests that the $D$. citri UAP genes are diverging rather rapidly. We have 
MVDINEGKDLLTSCNQQ-------------HLLKYWDEINDEEKS ILLNE---IKQLNIP -MDSTKSQDIFTSENSEIDPNKGRTLQKSLELEPNENVSKDKELNILLKDDVSLMNMDIF

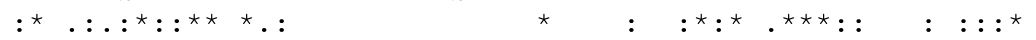

EACEYFKSANTYSRQAEAQCDSSMKPVPSELYGSAQDTSSDILKSYREIGLQEISEGHVG KARKYYEEACAVSNAPERFSEVQVFP-PNCLSG-VNTVDASTLGKYRELGLKLISRGDVA

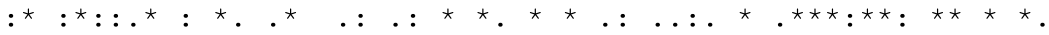

VILLAGGQGTRIGVPYPKGMYKVGLPSDKSLFQIQAERIMKLESLAFEQTGKKSIITWFI VIVLAGGQGTRLGADYPKGMYNIGLPSGKSLFQIQAEKIDKLIEIAKEKFG-SGCLPWFI

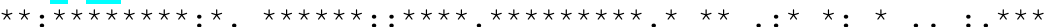

MTSESTMEPTKNFFEENKYFGLQKDNVIFFEQGVLPCFTFDGKI IMDSKFKIAKAPDGNG MTSELTDRPTREYFERNGYFGLDPAHVIFFKQRSMPCFSLSGEILLETRDRVARSPDGHG

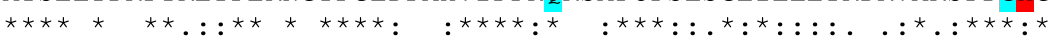

GIYIALKKKGILSEMEKRGIEYVHVYSVDNILVKVADPVFMGFCVKSQSDCGVKVVEKKL GLYHALGATGILDTMHTRGIKHIHVYCVDNILVKVGDPTFLGYCVEQGAHCGVKVVEKIT

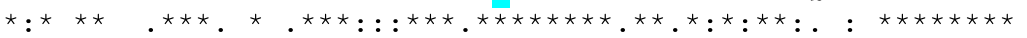

PNEGLGVVCVVDGQYKVVEYSEISSKTA-ELRDADGKLTFRAGNICNHFFSTAFLGQIAN PGESLGVLCNVDGKHKIVEYSELGNCSVFETQDQTGRLKFNLGSICNHYFSLECLQRMVK

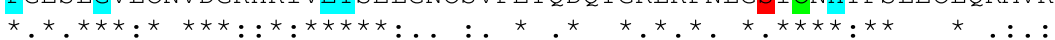

\section{DC_UAP1}

DC_UAP2

EHESKLKLHIAKKKIPYIDSKGLKVKPEQPNGIKIEKFIFDVFEFCKNLVVWEVAREHDF E-DAALKFHMARKKI PCLDEQGISQRPNKPNGIKLEKFLFDAFPLCENLVAWEVTRD-EF

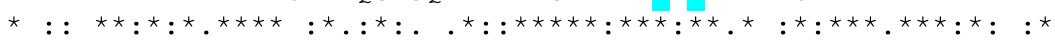

DC UAP1

DC_UAP2

SALKNSNAEK-TENPTTCCLALYDLHKSYIEAAGGTVKPDAVGNVVCEISPSVSYDGEGL SPLKNSPLDSASDNPVTCCQAVHALHARWIETAGGVVVADETGNTVCEIAPRVSYEGEGL

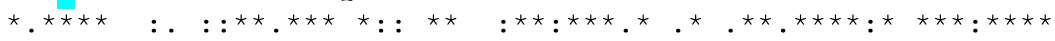

DC_UAP1

DC_UAP2
KPIVNGNTFESPILLK

EERVKGKVLQTPLLLE

$: \quad{ }^{\star}{ }^{*}: .:::^{\star}:{ }^{\star}$ :

Figure 5. Alignment of D. citri UAP1 and UAP2. Alignment was performed using MUSCLE (MUSCLE, RRID:SCR_011812) [17]. Individual amino acid alignments are denoted as identical (*), highly similar (:) or similar (.). Residues important for substrate binding by human UAP1 and conserved in T. castaneum are shaded according to their level of conservation. Identical residues are shaded blue and non-identical (but similar) residues are shaded red. The green shaded residue denotes the position of an alanine important for substrate binding in human UAP1 that is a cysteine in T. castaneum and other insects.

named the $D$. citri genes UAP1 and UAP2, but no implication is intended of direct orthology with duplicated UAP genes in other insects.

We compared available expression data from the two D. citri UAP genes using CGEN [19]. D. citri UAP1 is expressed in all tissues and stages examined, although expression levels vary (Figure 4). A few samples (e.g. female terminal abdomen and female leg) show high expression of UAP1, but these are single replicate samples that would need further verification. In the case of female terminal abdomen, single replicate data from a separate experiment shows only a moderate level of expression. Interestingly, D. citri UAP2 appears to show a sexually dimorphic expression pattern. It is expressed at a low-to-moderate level in most male tissues, with highest expression in abdominal samples, but shows little or no expression in the same tissues from females (Figures 4,6). While these observations are intriguing, the technical difficulty of creating RNA-seq libraries from miniscule amounts of dissected tissue, while maintaining the integrity of the RNA, in addition to the lack of statistical power provided by single replicate samples, mean that the expression data 


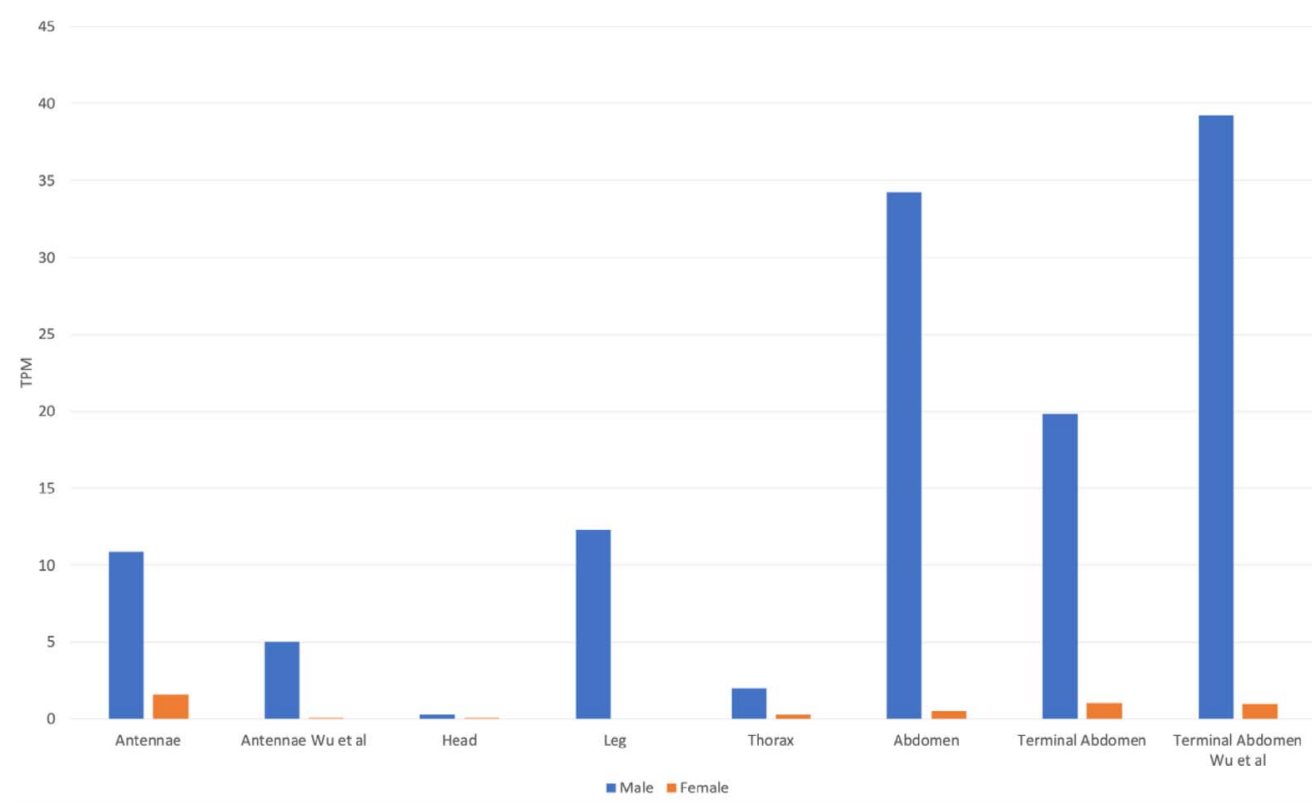

Figure 6. Expression levels of UAP2 in male and female tissues. Expression levels were obtained from the Citrus Greening Expression Network [19]. Tissue types are shown on the $X$ axis and expression levels (TPM) on the $Y$-axis. Blue bars denote expression levels in males and orange bars denote expression levels in females (all single replicate data). RNA-seq data from tissues labeled Wu et al. were sequenced in [22]. Data for the remaining tissues are from NCBI BioProject PRJNA448935.

currently available should be interpreted with caution. Experimental analysis is outside the scope of this data release, but additional studies of UAP1 and UAP2 expression and function in individual males and females will be necessary to verify these results.

\section{RE-USE POTENTIAL}

There is considerable interest in use of the genes described here as targets for pest control. At least two groups have already begun functional studies of the CHS gene in $D$. citri. Our improved annotations will allow more detailed experiments to be performed in the future. For example, isoform-specific RNAi experiments on the CHS gene could be designed to determine the function of each transcript variant. The revised gene models will be incorporated into a new official gene set, which will be available for BLAST analysis and expression profiling on the Citrus Greening website [42] and the CGEN [19].

\section{DATA AVAILABILITY}

The Diaphorina citri genome assembly, official gene sets, and transcriptome data are accessible via the Citrus Greening website [42]. All accessions for genes used for phylogenetic analysis are provided within this report, and all other data are available in the GigaScience GigaDB repository [43].

\section{EDITOR'S NOTE}

This article is one of a series of Data Releases crediting the outputs of a student-focused and community-driven manual annotation project curating gene models and if required, correcting assembly anomalies, for the Diaphorina citri genome project [2]. 


\section{DECLARATIONS}

\section{LIST OF ABBREVIATIONS}

CGEN: Citrus Greening Expression Network; CHS: chitin synthase; CLas: Candidatus Liberibacter asiaticus; NCBI: National Center for Biotechnology Information; OGS: Official Gene Set; RNAi: RNA interference; TPM: transcripts per million; UAP: UDP-N-acetylglucosamine pyrophosphorylase; UDP-GlcNAc: UDP-N-acetylglucosamine.

\section{ETHICAL APPROVAL}

Not applicable.

\section{CONSENT FOR PUBLICATION}

Not applicable.

\section{COMPETING INTERESTS}

The authors declare that they have no competing interests.

\section{FUNDING}

This work was supported by USDA-NIFA grant 2015-70016-23028, HSI 1300394, 2020-70029-33199 and an Institutional Development Award (IDeA) from the National Institute of General Medical Sciences of the National Institutes of Health under grant number P20GM103418.

\section{AUTHORS' CONTRIBUTIONS}

WBH, SJB, TD and LAM conceptualized the study; TD, SS, TDS and SJB supervised the study; SJB, TD, SS, and LAM contributed to project administration; SM, TDS, and BT conducted investigation; PH, MF-G, and SS contributed to software development; SS, TDS, PH, and MF-G developed methodology; SJB, TD, WBH, and LAM acquired funding; SM and TDS prepared and wrote the original draft; SS, WBH and SJB reviewed and edited the draft.

\section{ACKNOWLEDGEMENTS}

We thank Dr. Josh Benoit for assistance with data visualization.

\section{REFERENCES}

1 Zhu KY, Merzendorfer H, Zhang W, Zhang J, Muthukrishnan S, Biosynthesis, turnover, and functions of chitin in insects. Annu. Rev. Entomol., 2016; 61: 177-196.

2 Hosmani P et al. Chromosomal length reference assembly for Diaphorina citri using single-molecule sequencing and Hi-C proximity ligation with manually curated genes in developmental, structural and immune pathways. bioRxiv. 2019; 869685. https://doi.org/10.1101/869685.

3 Arakane $\mathbf{Y}$ et al. Characterization of two chitin synthase genes of the red flour beetle, Tribolium castaneum, and alternate exon usage in one of the genes during development. Insect Biochem. Mol. Biol., 2004; 34: 291-304.

4 Muthukrishnan S, Merzendorfer H, Arakane Y, Kramer KJ, Chitin metabolism in insects. In: Gilbert LI (ed.), Insect Molecular Biology and Biochemistry. Amsterdam: Elsevier 2012; pp. 193-235.

5 Wang $\mathbf{Y}$ et al. Chitin synthase 1 gene and its two alternative splicing variants from two sap-sucking insects, Nilaparvata lugens and Laodelphax striatellus (Hemiptera: Delphacidae). Insect Biochem. Mol. Biol., 2012; 42: 637-646.

6 Humphreys GB et al. Mummy, a UDP-N-acetylglucosamine pyrophosphorylase, modulates DPP signaling in the embryonic epidermis of Drosophila. Dev. Biol., 2013; 381: 434-445.

7 Adams MD et al. The genome sequence of Drosophila melanogaster. Science, 2000; 2185-2195. 
8 Holt RA et al. The genome sequence of the malaria mosquito Anopheles gambiae. Science, 2002; 298: 129-149.

9 Matthews BJ et al. Improved reference genome of Aedes aegypti informs arbovirus vector control. Nature, 2018; 563: 501-507.

10 Richards S et al. Tribolium Genome Sequencing Consortium. The genome of the model beetle and pest Tribolium castaneum. Nature, 2008; 452: 949-955.

11 Elsik CG et al. Finding the missing honey bee genes: lessons learned from a genome upgrade. BMC Genomics, 2014; 15: 86. doi:10.1186/1471-2164-15-86.

12 Werren JH et al. Functional and evolutionary insights from the genomes of three parasitoid Nasonia species. Science, 2010; 327: 343-348, doi:10.1126/science.1178028.

13 International Aphid Genomics Consortium. Genome sequence of the pea aphid Acyrthosiphon pisum. PLoS Biol., 2010; 8: e1000313. doi:10.1371/journal.pbio.1000313.

14 Chen W et al. The draft genome of whitefly Bemisia tabaci MEAM1, a global crop pest, provides novel insights into virus transmission, host adaptation, and insecticide resistance. BMC Biol., 2016; 14: 110. doi:10.1186/s12915-016-0321-y.

15 National Center for Biotechnology Information (NCBI). Ref-Seq Non-redundant Protein Database. Bethesda, MD: NCBI. https://www.ncbi.nlm.nih.gov/refseq/about/nonredundantproteins/. Accessed 27 October 2020.

16 Shippy TD et al. Annotating genes in Diaphorina citri genome version 3. protocols.io. 2020; http://doi.org/10.17504/protocols.io.bniimcce.

17 Madeira F et al. The EMBL-EBI search and sequence analysis tools APIs in 2019. Nucleic Acids Res., 2019; 47: W636-W641.

18 Larkin MA et al. Clustal W and Clustal X version 2.0. Bioinformatics, 2007; 23: 2947-2948.

19 Flores-Gonzalez $\mathbf{M}$ et al. Citrusgreening.org: an open access and integrated systems biology portal for the Huanglongbing (HLB) disease complex. bioRxiv. 2019; 868364. https://doi.org/10.1101/868364.

20 R Core Team. R: A language and environment for statistical computing. Vienna: R Foundation for Statistical Computing 2020; https://www.R-project.org/. Accessed December 172020.

21 Kolde R, pheatmap: Pretty Heatmaps (Version 1.0.12). 2020; https://cran.r-project.org/package=pheatmap.

22 Wu Z, Zhang H, Bin S, Chen L, Han Q, Lin J, Antennal and abdominal transcriptomes reveal chemosensory genes in the Asian Citrus Psyllid, Diaphorina citri. PLoS One, 2016; 11: e0159372.

23 Arakane Y et al. The Tribolium chitin synthase genes TcCHS1 and TcCHS2 are specialized for synthesis of epidermal cuticle and midgut peritrophic matrix. Insect Mol. Biol., 2005; 14: 453-463.

24 Arakane Y, Specht CA, Kramer KJ, Muthukrishnan S, Beeman RW, Chitin synthases are required for survival, fecundity and egg hatch in the red flour beetle, Tribolium castaneum. Insect Biochem. Mol. Biol., 2008; 38: 959-962.

25 Zhai Y et al. Identification and functional analysis of chitin synthase A in Oriental Armyworm, Mythimna separata. Proteomics, 2017; 17: doi:10.1002/pmic.201700165.

26 Mohammed AMA, DIab MR, Abdelsattar M, Khalil SMS, Characterization and RNAi-mediated knockdown of chitin synthase A in the potato tuber moth, Phthorimaea operculella. Sci. Rep., 2017; 7: 9502.

27 Shi J-F, Mu L-L, Chen X, Guo W-C, Li G-Q, RNA interference of chitin synthase genes inhibits chitin biosynthesis and affects larval performance in Leptinotarsa decemlineata (Say). Int. J. Biol. Sci., 2016; 12: 1319-1331.

28 Liu X, Cooper AMW, Yu Z, Silver K, Zhang J, Zhu KY, Progress and prospects of arthropod chitin pathways and structures as targets for pest management. Pestic. Biochem. Physiol., 2019; 161: 33-46.

29 Silva CP et al. Occurrence of midgut perimicrovillar membranes in paraneopteran insect orders with comments on their function and evolutionary significance. Arthropod. Struct. Dev., 2004; 33: 139-148.

30 Lu ZJ et al. Silencing of the chitin synthase gene is lethal to the Asian citrus psyllid, Diaphorina citri. Int J. Mol. Sci., 2019; 20: 3734. doi:10.3390/ijms20153734.

31 Edgar RC, MUSCLE: multiple sequence alignment with high accuracy and high throughput. Nucleic Acids Res., 2004; 32(5): 1792-1797, doi:10.1093/nar/gkh340. 
32 Kumar S, Stecher G, Li M, Knyaz C, Tamura K, MEGA X: Molecular Evolutionary Genetics Analysis across Computing Platforms. Mol. Biol. Evol., 2018; 35(6): 1547-1549, doi:10.1093/molbev/msy096.

33 Galdeano DM, Breton MC, Lopes JRS, Falk BW, Machado MA, Oral delivery of double-stranded RNAs induces mortality in nymphs and adults of the Asian citrus psyllid, Diaphorina citri. PLoS One, 2017; 12: e0171847.

34 Zhang X, Zhang J, Park Y, Zhu KY, Identification and characterization of two chitin synthase genes in African malaria mosquito, Anopheles gambiae. Insect. Biochem. Mol. Biol., 2012; 42: 674-682.

35 Hogenkamp DG et al. Chitin synthase genes in Manduca sexta: characterization of a gut-specific transcript and differential tissue expression of alternately spliced mRNAs during development. Insect. Biochem. Mol. Biol., 2005; 35: 529-540.

36 Araújo SJ, Aslam H, Tear G, Casanova J, mummy/cystic encodes an enzyme required for chitin and glycan synthesis, involved in trachea, embryonic cuticle and CNS development - Analysis of its role in Drosophila tracheal morphogenesis. Dev. Biol., 2005; 288: 179-193.

37 Tonning A, Helms S, Schwarz H, Uv AE, Moussian B, Hormonal regulation of mummy is needed for apical extracellular matrix formation and epithelial morphogenesis in Drosophila. Development, 2006; 133: 331-341.

38 Schimmelpfeng K, Strunk M, Stork T, Klämbt C, mummy encodes an UDP-N-acetylglucosamine-dipohosphorylase and is required during Drosophila dorsal closure and nervous system development. Mech. Dev., 2006; 123: 487-499.

39 Arakane $\mathrm{Y}$ et al. Both UDP N-acetylglucosamine pyrophosphorylases of Tribolium castaneum are critical for molting, survival and fecundity. Insect. Biochem. Mol. Biol., 2011; 41: 42-50.

40 Liu X et al. Molecular and functional analysis of UDP-N-acetylglucosamine pyrophosphorylases from the migratory locust, Locusta migratoria. PLoS One, 2013; 8: e71970.

41 Shi JF, Fu J, Mu LL, Guo WC, Li GQ, Two Leptinotarsa uridine diphosphate N-acetylglucosamine pyrophosphorylases are specialized for chitin synthesis in larval epidermal cuticle and midgut peritrophic matrix. Insect. Biochem. Mol. Biol., 2016; 68: 1-12.

42 Citrus Greening Solutions. Annotation of psyllid genome. 2018;

https://citrusgreening.org/annotation/index. Accessed 18 December 2020.

43 Miller S, Shippy TD, Tamayo B, Hosmani PS, Flores-Gonzalez M, Mueller LA, Hunter WB, Brown SJ, D'Elia T, Saha S, Supporting data for "Annotation of chitin biosynthesis genes in Diaphorina citri, Asian citrus psyllid”. GigaScience Database. 2021; http://dx.doi.org/10.5524/100900. 